\title{
The Landau-Lifshitz Equation, Elliptic Curves and the Ward Transform
}

\author{
A.L. Carey ${ }^{1}$, K.C. Hannabuss ${ }^{2}$, L.J. Mason ${ }^{3}$, and M.A. Singer ${ }^{4}$ \\ ${ }^{1}$ Department of Pure Mathematics, University of Adelaide, South Australia 5001 \\ ${ }^{2}$ Balliol College, Oxford, U.K. \\ ${ }^{3}$ Mathematical Institute, Oxford, U.K. \\ ${ }^{4}$ Lincoln College, Oxford, U.K.
}

Received September 8, 1992

\begin{abstract}
The Landau-Lifshitz (LL) equation is studied from a point of view that is close to that of Segal and Wilson's work on KdV. The LL hierarchy is defined and shown to exist using a dressing transformation that involves parameters $\lambda_{1}, \lambda_{2}, \lambda_{3}$ that live on an elliptic curve $\Sigma$. The crucial role of the group $K \simeq \mathbb{Z}_{2} \times \mathbb{Z}_{2}$ of translations by the half-periods of $\Sigma$ and its non-trivial central extension $\tilde{K}$ is brought out and an analogue of Birkhoff factorisation for $\tilde{K}$-equivariant loops in $\Sigma$ is given. This factorisation theorem is given two treatments, one in terms of the geometry of an infinite-dimensional Grassmannian, and the other in terms of the algebraic geometry of bundles over $\Sigma$. Further, a Ward-like transform between a class of holomorphic vector bundles on the total space $Z$ of a line-bundle over $\Sigma$ and solutions of LL is constructed. An appendix is devoted to a careful definition of the Grassmannian of the Frechet space $C^{\infty}\left(S^{1}\right)$.
\end{abstract}

\section{Introduction}

This paper aims to expose the links between a variety of methods for solving completely integrable non-linear equations in the special case of the LandauLifshitz (LL) equation (cf. (2.23)). The interest in this example stems from the fact that the spectral curve $\Sigma$ which arises from the Lax form of the equations is an elliptic curve. This means [13] that there is no immediate generalisation of the methods which are applicable when the spectral curve is the Riemann sphere. For example, a generic $\mathrm{SL}_{2}(\mathbb{C})$-valued loop on the unit circle has a Birkhoff factorisation as a product of loops, one holomorphic inside the unit disc, the other holomorphic outside the disc. There is however no such factorisation in general for a disc in $\Sigma$. It emerges that the only loops that arise in the study of the LL equation behave in a prescribed fashion under a discrete group of symmetries of $\Sigma$ and for such loops an appropriate analogue of Birkhoff factorisation does exist (cf. Theorems 3.1 and 4.1 below).

Previous literature on the LL equation may be traced from [7]. Our motivation for its study stemmed partly from the relation to conformal field theory that is 
revealed by the approach of the Kyoto school [5]. In [2], a framework was developed (see also the Appendix) for extending the methods of [14] for the KdV equation to the case of LL. These are used in Sect. 3 to prove one version of the factorisation theorem mentioned above.

In Sect. 4 we give a more geometric version of our factorisation theorem which rests on Atiyah's classification of vector bundles over elliptic curves [1].

In Sect. 5, a twistorial description of LL is given along the lines of [8] and [9].

We close with two further remarks. First, and this is another reason for its interest, LL is an example of an integrable system which is not in any obvious way a reduction of the self-dual Yang-Mills equations [15]. Nonetheless, methods very similar to those of [8] are applicable to its study. Secondly, LL points the way to the analysis of integrable systems with higher-genus spectral curves.

\section{Preliminaries}

2.1. Background on Elliptic Curves. Fix $\Sigma=\mathbb{C} / \Lambda$, an elliptic curve. $\Lambda$ is the lattice generated by 1 and $\tau(\operatorname{Im} \tau>0)$ and $z$ is a complex parameter on $\mathbb{C}$. Let $K$ be the Klein 4-group of half-period automorphisms of $\Sigma$, so $K$ consists of the elements

$$
z \mapsto z, \quad z \mapsto z+1 / 2, \quad z \mapsto z+\tau / 2, \quad z \mapsto z+1 / 2+\tau / 2 .
$$

We often refer to the second of these as $\#$ and to the third as $b$. Important also will be the non-trivial central extension $\tilde{K}$ of $K$ by $\mathbb{Z}_{2}$. This has an explicit realization in terms of Pauli matrices,

$$
\tilde{K}=\left\{ \pm 1, \pm i \sigma_{1}, \pm \sigma_{2}, \pm \sigma_{3}\right\}
$$

where

$$
\sigma_{1}=\left[\begin{array}{ll}
0 & 1 \\
1 & 0
\end{array}\right], \quad \sigma_{2}=\left[\begin{array}{rr}
0 & -i \\
i & 0
\end{array}\right], \quad \sigma_{3}=\left[\begin{array}{rr}
1 & 0 \\
0 & -1
\end{array}\right] .
$$

Definition. If $U$ is a $K$-invariant subset of $\Sigma$, we shall write $\mathscr{G}_{U}$ for the group of holomorphic maps $\gamma: U \rightarrow \mathrm{SL}_{2}(\mathbb{C})$ that are $K$-equivariant in the sense that:

$$
\gamma(z+1 / 2)=\sigma_{2} \gamma(z) \sigma_{2}^{-1}
$$

and

$$
\gamma(z+\tau / 2)=\sigma_{3} \gamma(z) \sigma_{3}^{-1} .
$$

We note that $\gamma$ lies in $\mathscr{G}_{U}$ if and only if

$$
\gamma(z)=\left[\begin{array}{rr}
f(z) & -g^{\sharp}(z) \\
g(z) & f^{\sharp}(z)
\end{array}\right],
$$

where the functions $f, g, f^{\#}, g^{\#}$ are holomorphic on $U$ and satisfy

$$
f^{\sharp}(z)=f(z+1 / 2), \quad g^{\#}(z)=g(z+1 / 2) ;
$$

and

$$
f(z+\tau / 2)=f(z), \quad g(z+\tau / 2)=-g(z) .
$$

In order to understand $\mathscr{G}_{U}$ better, we shall need a good understanding of the meromorphic functions on $\Sigma$. To this end, we proceed as in Chapter 1, Sects. 1-6 
of [11]. Introduce first the $\vartheta$-functions

$$
\vartheta_{a b}(z)=\sum_{n \in \mathbb{Z}} \mathbf{e}\left[\tau(n+a / 2)^{2} / 2+(n+a / 2)(z+b / 2)\right],
$$

where $\mathbf{e}(u)=\exp (2 \pi i u)$ and

$$
(a, b)=(0,0),(1,0),(0,1),(1,1) .
$$

One has

$$
\vartheta_{a b}(z+1)=(-1)^{a} \vartheta_{a b}(z), \quad \vartheta_{a b}(z+\tau)=(-1)^{b} \mathbf{e}(-\tau / 2-z) \vartheta_{a b}(z) .
$$

The zeros of $\vartheta_{a b}$ are as follows:

Put

$$
\begin{aligned}
& \left\{z: \vartheta_{00}(z)=0\right\}=\Lambda+1 / 2+\tau / 2 ; \\
& \left\{z: \vartheta_{10}(z)=0\right\}=\Lambda+1 / 2 ; \\
& \left\{z: \vartheta_{01}(z)=0\right\}=\Lambda+\tau / 2 ; \\
& \left\{z: \vartheta_{11}(z)=0\right\}=\Lambda .
\end{aligned}
$$

$$
\lambda_{1}(z)=\frac{\vartheta_{00}(2 z)}{\vartheta_{00} \vartheta_{11}(2 z)}, \quad \lambda_{2}(z)=\frac{\vartheta_{10}(2 z)}{\vartheta_{10} \vartheta_{11}(2 z)}, \quad \lambda_{3}(z)=\frac{\vartheta_{01}(2 z)}{\vartheta_{01} \vartheta_{11}(2 z)},
$$

where for $(a, b) \neq(1,1), \vartheta_{a b}=\vartheta_{a b}(0)$. It follows from the quasi-periodicity (2.8) of the $\vartheta_{a b}$ that the $\lambda_{i}$ are $\Lambda$-periodic meromorphic functions on $\mathbb{C}$ and hence represent meromorphic functions on $\Sigma$.

For future use we note that the behaviour of the $\lambda_{i}$ under $K$ is given by the following table:

\begin{tabular}{lrr}
\hline$z$ & $z+1 / 2$ & $z+\tau / 2$ \\
\hline$\lambda_{1}$ & $-\lambda_{1}$ & $-\lambda_{1}$ \\
$\lambda_{2}$ & $\lambda_{2}$ & $-\lambda_{2}$ \\
$\lambda_{3}$ & $-\lambda_{3}$ & $\lambda_{3}$ \\
\hline
\end{tabular}

We also note Riemann's identities (p. 23, Eqs. $E_{1}, E_{2}, M_{1}$ of [11]) for the $\lambda_{i}$ :

$$
\begin{aligned}
& \lambda_{1}^{2}-\lambda_{2}^{2}=\vartheta_{01}^{2} / \vartheta_{00}^{2} \vartheta_{10}^{2}=J_{1}-J_{2} ; \\
& \lambda_{2}^{2}-\lambda_{3}^{2}=-\vartheta_{00}^{2} / \vartheta_{01}^{2} \vartheta_{10}^{2}=J_{2}-J_{3} ; \\
& \lambda_{3}^{2}-\lambda_{1}^{2}=\vartheta_{10}^{2} / \vartheta_{00}^{2} \vartheta_{01}^{2}=J_{3}-J_{1} ;
\end{aligned}
$$

(these equations serving to define the $J_{i}$ to within an overall additive constant) and

$$
\vartheta_{00}^{4}=\vartheta_{01}^{4}+\vartheta_{10}^{4} \text {. }
$$

The $\lambda_{i}$ define a holomorphic embedding of what we shall call the finite part of $\Sigma$, namely $\Sigma-\{0,1 / 2, \tau / 2,1 / 2+\tau / 2\}$ in $\mathbb{C}^{3}$; Riemann's identities indicate that the image under this embedding is an intersection of quadric surfaces. Note that we shall often call the points $0,1 / 2, \tau / 2,1 / 2+\tau / 2$ the infinity of $\Sigma$. 
The following results are devoted to establishing convenient versions of the notion of Laurent series expansion on $\Sigma$. Let $\mathscr{C}$ be the union of circles of radius $\varepsilon$ about the four points at infinity $-0,1 / 2, \tau / 2,1 / 2+\tau / 2$ (Fig. 1). We suppose that $\varepsilon$ is so small that these circles are disjoint; it follows that none of the $\lambda_{i}$ has a pole or zero on $\mathscr{C}$.

Lemma 2.1. Let $f$ be $a\left(C^{\infty}\right.$ or $\left.C^{\omega}\right) \mathbb{C}$-valued function on $\mathscr{C}$ which satisfies a periodicity condition of the form

$$
f(z+1 / 2)= \pm f(z), \quad f(z+\tau / 2)= \pm f(z) .
$$

Then there exist unique complex numbers $f_{n}$ such that, on $\mathscr{C}$,

$$
\begin{array}{ll}
f=\sum f_{2 k} \lambda_{3}^{2 k}+\sum f_{2 k+1} \lambda_{1} \lambda_{2} \lambda_{3}^{2 k-1}, & (++) \\
f=\sum f_{2 k} \lambda_{1} \lambda_{2} \lambda_{3}^{2 k-2}+\sum f_{2 k+1} \lambda_{3}^{2 k+1}, & (-+) \\
f=\sum f_{2 k} \lambda_{1} \lambda_{3}^{2 k-1}+\sum f_{2 k+1} \lambda_{2} \lambda_{3}^{2 k}, & (+-) \\
f=\sum f_{2 k} \lambda_{2} \lambda_{3}^{2 k-1}+\sum f_{2 k+1} \lambda_{1} \lambda_{3}^{2 k}, & (--) .
\end{array}
$$

Proof. Expand $f$ on $|z|=\varepsilon$ as a Fourier series $\sum F_{n} e^{i n \theta}$. The periodicity conditions determine $f$ on the rest of $\mathscr{C}$. Consider the case $(++)$ and put $u_{2 k}=\lambda_{3}^{2 k}$, $u_{2 k+1}=\lambda_{1} \lambda_{2} \lambda_{3}^{2 k-1}$. Then $u_{n}$ has a pole of order $n$ at $z=0$ (interpreted as a zero of order $-n$ if $n<0)$ and so, restricting to $|z|=\varepsilon$, the $\mathbb{Z} \times \mathbb{Z}$ matrix relating $\left\{e^{-i n \theta}\right\}$ and $\left\{u_{n}\right\}$ is invertible (in fact, triangular). Thus the Fourier expansion is equivalent to the claimed expansion in the $u_{n}$, on $|z|=\varepsilon$. Since the $u_{n}$ have been chosen to have the same periodicity property as $f$, the expansion holds also on the rest of $\mathscr{C}$.

The proof for the other cases is the same.

Note that Riemann's identities (2.10) give linear dependences between $\lambda_{1}^{2}$, $\lambda_{2}^{2}$ and $\lambda_{3}^{2}$, so other expansion schemes are possible.

A simple consequence which has obvious application to the study of the group $\mathscr{G}_{U}$ defined above, is the following:

Corollary 2.2. Let $f, g$ be $\left(C^{\infty}\right.$ or $\left.C^{\omega}\right) \mathbb{C}$-valued functions on $\mathscr{C}$ which obey (2.5):

$$
f(z+\tau / 2)=f(z), g(z+\tau / 2)=-g(z) .
$$

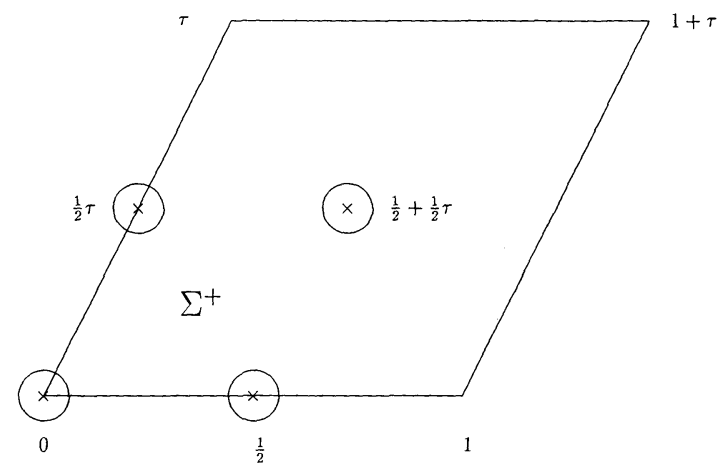

Fig. 1. The torus $\Sigma$ with its points at $\infty$ and the curve $\mathscr{C}$ 
Then $f, g$ have unique expansions in the form

$$
f=\sum\left(f_{n} \lambda_{3}^{n}+\tilde{f}_{n} \lambda_{1} \lambda_{2} \lambda_{3}^{n-2}\right)
$$

and

$$
g=\sum\left(g_{n} \lambda_{2} \lambda_{3}^{n-1}+\tilde{g}_{n} \lambda_{1} \lambda_{3}^{n-1}\right)
$$

Example. If $A$ is $K$-equivariant and polynomial of degree 1 in the $\lambda_{i}$, then $A$ has the form

$$
A=a_{0}+i\left(b_{1} \lambda_{1} \sigma_{1}+c_{1} \lambda_{2} \sigma_{2}+d_{1} \lambda_{3} \sigma_{3}\right)
$$

for some constants $a_{0}, b_{1}, c_{1}, d_{1}$. If $B$ is $K$-equivariant and polynomial of degree 2 in the $\lambda_{i}$ then $B$ has the form

$$
B=a_{0}+a_{2} \lambda_{3}^{2}+i\left(b_{1} \lambda_{1}+b_{2} \lambda_{2} \lambda_{3}\right) \sigma_{1}+\left(c_{1} \lambda_{2}+c_{2} \lambda_{1} \lambda_{3}\right) \sigma_{2}+\left(d_{1} \lambda_{3}+d_{2} \lambda_{1} \lambda_{2}\right) \sigma_{3},
$$

for some further constants $a_{2}, b_{2}, c_{2}, d_{2}$.

2.2. The LL Hierarchy. We proceed to give an algebraic description of the LL hierarchy in a fashion analogous to the scheme of Drinfeld-Sokolov [6] for hierarchies of $\mathrm{KdV}$ type. The key idea is that of "dressing."

Definition. Let $\mathscr{S}$ be the space of connections on the interval $a \leqq x \leqq b$ of the form

$$
\partial_{x}-A
$$

where the $2 \times 2$ matrix

$$
A=i\left(\lambda_{1} S_{1} \sigma_{1}+\lambda_{2} S_{2} \sigma_{2}+\lambda_{3} S_{3} \sigma_{3}\right)
$$

is K-equivariant, polynomial of degree 1 in the $\lambda_{i}$, trace-free and normalized so that

$$
S_{1}^{2}+S_{2}^{2}+S_{3}^{2}=1 \text {. }
$$

$\mathscr{S}$ is called the space of LL initial data. A LL flow of order $r$ on $\mathscr{S}$ is an evolution of the form

$$
\left[\partial_{t}-B, \partial_{x}-A\right]=0
$$

where $B$ is $K$-equivariant, polynomial of degree $r$ in the $\lambda_{i}$ and trace-free.

To prove the existence of the LL hierarchy, that is, the existence of mutually commuting LL-flows of each order, we begin with the following dressing theorem.

Theorem 2.3. Given any element

$$
\partial_{x}-i\left(\lambda_{1} S_{1} \sigma_{1}+\lambda_{2} S_{2} \sigma_{2}+\lambda_{3} S_{3} \sigma_{3}\right)
$$

of $\mathscr{S}$, there exists a K-equivariant $S L_{2}(\mathbb{C})$ gauge transformation $\gamma$, of the form given below, such that

$$
\gamma \cdot\left(\partial_{x}-i \lambda_{3} \sigma_{3}\right) \cdot \gamma^{-1}=\partial_{x}-i\left(\lambda_{1} S_{1} \sigma_{1}+\lambda_{2} S_{2} \sigma_{2}+\lambda_{3} S_{3} \sigma_{3}\right)
$$

If we write

$$
\gamma=a-i\left(b \sigma_{1}+c \sigma_{2}+d \sigma_{3}\right)
$$


then $a, b, c, d$ are required to have formal series expansions:

$$
\begin{aligned}
& a=\sum_{k \leqq 0}\left(a_{2 k} \lambda_{3}^{2 k}+a_{2 k-1} \lambda_{1} \lambda_{2} \lambda_{3}^{2 k-3}\right), \\
& b=\sum_{k \leqq 0}\left(b_{2 k} \lambda_{2} \lambda_{3}^{2 k-1}+b_{2 k-1} \lambda_{1} \lambda_{3}^{2 k-2}\right), \\
& c=\sum_{k \leqq 0}\left(c_{2 k} \lambda_{1} \lambda_{3}^{2 k-1}+c_{2 k-1} \lambda_{2} \lambda_{3}^{2 k-2}\right), \\
& d=\sum_{k \leqq 0}\left(d_{2 k} \lambda_{1} \lambda_{2} \lambda_{3}^{2 k-2}+d_{2 k-1} \lambda_{3}^{2 k-1}\right) .
\end{aligned}
$$

Proof. Multiply the given Eq. (2.14) on the right by $\gamma$, substitute for $\gamma$ using (2.15) and (2.16), and collect coefficients of the various powers of the $\lambda_{i}$. The result is the following system of equations:

$$
M \phi_{n}=-\partial_{x} \phi_{n+1}+\psi_{n+2}, \quad(n \leqq 0),
$$

where

$$
M=\left[\begin{array}{cccc}
0 & -S_{1} & -S_{2} & 1-S_{3} \\
S_{1} & 0 & -1-S_{3} & S_{2} \\
S_{2} & 1+S_{3} & 0 & -S_{1} \\
-1+S_{3} & -S_{2} & S_{1} & 0
\end{array}\right],
$$

$\phi_{n}=\left[a_{n}, b_{n}, c_{n}, d_{n}\right]^{t}$ and $\psi_{n}$ is a linear function of $\phi_{n}$ (which depends also upon the $S_{i}$ and the $J_{i j}$ ). (Here we have put $a_{n}, b_{n}, c_{n}, d_{n}$ equal to 0 for $n>0$.)

Note that the determinant of $M$ is equal to $\left(1-S_{1}^{2}-S_{2}^{2}-S_{3}^{2}\right)^{2}$ and so $M$ has rank 2 if and only if the $S_{i}$ are normalized as in the definition of $\mathscr{S}$ above. With this condition assumed to hold, we have the decomposition $\mathbb{C}^{4}=I \oplus Q$, where $I$ is the image of $M$ and $Q$ is the kernel of $M$; and $\operatorname{dim} I=\operatorname{dim} Q=2$.

To solve (2.17) we proceed inductively. When $n=0$, the RHS $=0$ and so $(2.17)_{0}$ is solved by any $\phi_{0}$ in $Q$.

The inductive assumption is that for some $n<-1$, we have found $\phi_{0}, \phi_{-1}, \ldots, \phi_{n+1}$ and $\phi_{n}$, the latter up to the addition of any element of $Q$. The inductive step has two parts. Fix a choice $\tilde{\phi}_{n}$ of $\phi_{n}$ so that all other choices have the form $\tilde{\phi}_{n}+u$ for some $u$ in $Q$. Choose $u$ so as to make $(2.17)_{n-1}$ solvable, by solving the ODE

$$
\Pi_{Q}\left(\partial_{x} u+\partial_{x} \tilde{\phi}_{n}-\psi_{n+1}\right)=0
$$

where we have written $\Pi_{Q}$ for the projection onto $Q$. The second part of the inductive step is to solve the resulting linear equation for $\phi_{n-1}$. The inductive step is completed by observing that this solution is determined up to the addition of any element of $Q$.

We now have a formal solution of our Eq. (2.14) which, may, however, not have unit determinant. To rectify this, take the trace of (2.14):

$$
\operatorname{tr}\left(\partial_{x} \gamma \cdot \gamma^{-1}\right)=\partial_{x} \log \operatorname{det} \gamma=0 .
$$

Thus $\operatorname{det} \gamma$ is a constant $C \neq 0$ and by multiplying by $C^{-1 / 2}$ we get a gauge transformation $\gamma$ with all the properties required by the theorem. 
To construct the LL flows on $\mathscr{S}$, put

$$
U_{2 k}=i \lambda_{1} \lambda_{2} \lambda_{3}^{2 k-2} \sigma_{3}, \quad U_{2 k-1}=i \lambda_{3}^{2 k-1} \sigma_{3} .
$$

Then for each $r, U_{r}$ is $K$-equivariant and polynomial of degree $r$; moreover,

$$
\left[\partial_{r}-U_{r}, \partial_{s}-U_{s}\right]=0 \text { for } r, s=1,2, \ldots
$$

Here we have introduced the infinitely many time variables $t_{1}=x, t_{2}, t_{3}, \ldots$ and have written $\partial_{r}$ for $\partial / \partial t_{r}$.

Now given $\partial_{x}-A$ in $\mathscr{S}$, construct the gauge transformation $\gamma$ of Theorem 2.3 and for each $r$, put

$$
\partial_{r}-B_{r}=\gamma \cdot\left(\partial_{r}-U_{r}\right) \cdot \gamma^{-1}
$$

choosing the (hitherto undefined) dependence of $\gamma$ upon $t_{r}$ so that $B_{r}$ is polynomial in the $\lambda_{i}$. Then $A=B_{1}$ and

$$
\left[\partial_{r}-B_{r}, \partial_{s}-B_{s}\right]=0 \text { for } r, s=1,2, \ldots
$$

is a formal consequence of (2.19) and the fact that for each $r, \partial_{r}-B_{r}$ is gauge equivalent to $\partial_{r}-U_{r}$ (Eq. (2.20)).

We have to check finally that the flows constructed by this method preserve the normalization of the $S_{i}$ given in the definition of $\mathscr{S}$. To do so, multiply the evolution equation got by putting $s=1$ in (2.21) by $A=B_{1}$ and take the trace:

$$
\operatorname{tr}\left(A \partial_{r} A\right)=\operatorname{tr}\left(B_{1} \partial_{1} B_{r}\right) .
$$

The LHS of this equation is equal to

$$
-\lambda_{3}^{2} \partial_{r}\left(S_{1}^{2}+S_{2}^{2}+S_{3}^{2}\right)-2\left(J_{13} S_{1} \partial_{r} S_{1}+J_{23} S_{2} \partial_{r} S_{2}\right)
$$

so we will be done if we can prove that the coefficient of $\lambda_{3}^{2}$ on the RHS is zero. Using the formula (2.20) for $B_{1}$ and $B_{r}$, we calculate that the RHS of (2.22) is equal to

$$
\operatorname{tr}\left[\left(\partial_{1} \gamma \cdot \gamma^{-1}+\gamma U_{1} \gamma^{-1}\right) \partial_{1}\left(\partial_{r} \gamma \cdot \gamma^{-1}+\gamma U_{r} \gamma^{-1}\right)\right]
$$

The part of this that could contribute to the coefficient of $\lambda_{3}^{2}$ is

$$
\operatorname{tr}\left[\left(\partial_{1} \gamma \cdot \gamma^{-1}+\gamma U_{1} \gamma^{-1}\right) \partial_{1}\left(\gamma U_{r} \gamma^{-1}\right)\right]
$$

It is easy to check that this vanishes.

To summarise the discussion of this section: we have introduced the spectral curve $\Sigma$ for the Lax form of LL and the group $K$ of symmetries. We have also introduced the space $\mathscr{S}$ of LL initial data and defined a hierarchy of commuting flows on $\mathscr{S}$. We have shown that such flows exist by the use of a dressing transformation. We close by writing down $B_{2}$, the matrix which defines the basic LL flow, leaving the energetic reader to derive it by the methods of this section.

Example. If as before,

$$
A=i\left(\lambda_{1} S_{1} \sigma_{1}+\lambda_{2} S_{2} \sigma_{2}+\lambda_{3} S_{3} \sigma_{3}\right),
$$

then we claim that

$$
B_{2}=i\left[\left(\lambda_{2} \lambda_{3} T_{1}+\lambda_{1} U_{1}\right) \sigma_{1}+\left(\lambda_{1} \lambda_{3} T_{2}+\lambda_{2} U_{2}\right) \sigma_{2}+\left(\lambda_{1} \lambda_{2} T_{3}+\lambda_{3} U_{3}\right) \sigma_{3}\right],
$$


where

$$
\mathbf{T}=\mathbf{S} \quad \text { and } \quad \mathbf{U}=\mathbf{S} \wedge \mathbf{S}_{x}
$$

The condition

$$
\left[\partial_{x}-A, \partial_{t}-B_{2}\right]=0
$$

can be checked to be equivalent to the LL equation

$$
\mathbf{S}_{t}=\mathbf{S} \wedge \mathbf{S}_{x x}+\mathbf{S} \wedge J \mathbf{S} .
$$

where $J=\operatorname{diag}\left(J_{1}, J_{2}, J_{3}\right)$.

\section{An Analogue of Birkhoff Factorisation for Elliptic Curves}

In this section, we present the first version of our factorisation for $K$-equivariant loops. The method of proof involves the infinite-dimensional geometry of a certain Grassmannian and is analogous to the proof of Birkhoff factorisation given in [12]. These methods give a rigorous framework for the conformal field theoretic approach of [5]. This point of view is briefly discussed here also.

As in Sect. 2.1, we let $\mathscr{C}$ be the union of the four circles of radius $\varepsilon$ about the points $0,1 / 2, \tau / 2,1 / 2+\tau / 2$ in $\Sigma$, where $\varepsilon$ is chosen so small that these circles are disjoint. We denote by $\Sigma_{+}\left(\Sigma_{-}\right)$the region outside $\mathscr{C}$ (resp. inside $\mathscr{C}$ ) including the boundary (Fig. 1).

Definition. We denote by $\mathscr{G}$ the group of smooth maps $\gamma: \mathscr{C} \rightarrow S L_{2}(\mathbb{C})$ such that $\gamma$ is $K$-equivariant. We denote by $\mathscr{G}_{ \pm}$the subgroup of $\mathscr{G}$ whose entries consist of boundary values of $K$-equivariant holomorphic maps: $\Sigma_{ \pm} \rightarrow S L_{2}(\mathbb{C})$.

We note that the definition makes sense because $\mathscr{C}$ is $K$-invariant and recall the description of elements of $\mathscr{G}$ that is contained in Eqs. (2.1)-(2.5) above.

The main result of this section is the following factorisation theorem for elements of $\mathscr{G}$.

Theorem 3.1. Let $\mathscr{G}, \mathscr{G}_{ \pm}$be as above. Then there is a dense open set $\mathscr{G}_{0}$ of $\mathscr{G}$ such that if $\gamma \in \mathscr{G}_{0}$, there exist $\gamma_{ \pm} \in \mathscr{G}_{ \pm}$unique up to overall sign such that $\gamma=\gamma_{+}^{-1} \gamma_{-}$.

Remark. (1) The plausibility of this result can be checked by considering its "infinitesimal version." This states that for any element $A$ of the Lie algebra of $\mathscr{G}$, there is a unique splitting $A=A_{+}+A_{-}$, where $A_{ \pm}$is a $K$-equivariant holomorphic map from $\Sigma_{ \pm}$into the Lie algebra of $\mathrm{SL}_{2}(\mathbb{C})$. This is a consequence of Corollary 2.2. Write $A$ in terms of $f$ and $g$ and expand them according to (2.12) and (2.13). Because $\operatorname{tr}(A)=0$, we have

$$
f_{0}=\tilde{f}_{ \pm 1}=f_{ \pm 2}=\cdots=0 .
$$

Thus the required splitting of $A$ corresponds to $f=f_{+}+f_{-}, g=g_{+}+g_{-}$, where $f_{+}$ (resp. $g_{+}$) is given by the expansion (2.12) (resp. (2.13)), the sum extending over $n>0$, and $f_{-}=f-f_{+}, g_{-}=g-g_{+}$.

(2) If $\gamma$ in $\mathscr{G}_{0}$ is a real-analytic element, this theorem asserts the triviality of the holomorphic vector bundle $E_{\gamma}$ over $\Sigma$ obtained by using $\gamma$ as a clutching function. A precise statement of this geometric version of the result appears below (Theorem 4.1). It follows from that result, combined with Proposition 4.3, that $\mathscr{G}_{0}=\mathscr{G}$. 
Proof. We mimic the proof of Birkhoff factorisation that is given in [12]. Let

$$
\mathscr{V}=\left\{u \in C^{\infty}\left(\mathscr{C}, \mathbb{C}^{2}\right) \mid u(z+\tau / 2)=\sigma_{3} u(z)\right\}
$$

and introduce a polarisation $\mathscr{V}=\mathscr{V}_{+} \oplus \mathscr{V}_{-}$as follows. If $[f, g]^{t}$ is in $\mathscr{V}$, then $f(z+\tau / 2)=f(z)$ and $g(z+\tau / 2)=-g(z)$. So we can expand $f$ and $g$ according to Corollary 2.2. Define $g_{+}$by letting the sum in (2.13) run over all $n>0$ and put $g_{-}=g-g_{+}$. Then $g_{ \pm}$is holomorphic in $\Sigma_{ \pm}$.

We cannot polarise $f$ so cleanly: there is ambiguity arising from the term in $f_{0}$ (which is holomorphic in both $\Sigma_{ \pm}$) and the term in $\tilde{f}_{1}$ (which is holomorphic in neither). We proceed by observing that the function $\left(\lambda_{1}-\lambda_{2}\right) f$ has the same behaviour under $b$ as does $g$ and can be split accordingly. (This particular resolution of the ambiguities follows the approach to the polarisation given in [2].) To make this explicit, rewrite the expansion (2.12) as

$$
f=\sum f_{n}^{\prime} \lambda_{3}^{n}+\sum_{n \neq 1} \tilde{f}_{n} \lambda_{1} \lambda_{2} \lambda_{3}^{n-2}+\tilde{f}_{1}^{\prime}\left(\lambda_{3}+\lambda_{1} \lambda_{2} \lambda_{3}^{-1}\right)
$$

put

$$
f_{+}=\sum_{n \geqq 0} f_{n}^{\prime} \lambda_{3}^{n}+\sum_{n \geqq 2} \tilde{f}_{n} \lambda_{1} \lambda_{2} \lambda_{3}^{n-2}
$$

and $f_{-}=f-f_{+}$.

Then $\mathscr{V}_{+}$consists of those functions holomorphic on $\Sigma_{+}$but $\mathscr{V}_{-}$consists of those functions which are holomorphic on $\Sigma_{-}-\{0, \tau / 2\}$, with at worst a simple pole at $\{0, \tau / 2\}$, modulo the subspace $\mathbb{C} \cdot[1,0]^{t}$.

This polarisation defines a Grassmannian $\mathrm{Gr}_{\text {tame }}$ as in the Appendix and $\mathscr{G}$ acts on $\mathscr{V}$ and on $\mathrm{Gr}_{\text {tame }}$. Define $\mathscr{G}_{0} \subset \mathscr{G}$ as the set of $\gamma$ for which the transversality condition $\gamma\left(\mathscr{V}_{+}\right) \cap \mathscr{V}_{-}=0$ holds. For $\gamma \in \mathscr{G}_{0}$, there exists an operator $T_{\gamma}: \mathscr{V}_{+} \rightarrow \mathscr{V}_{-}$ such that $\gamma\left(\mathscr{V}_{+}\right)$is the graph of $T_{\gamma}$.

Since $[1,0]^{t}$ lies in $\mathscr{V}_{+}$, we may put $f=\left(\begin{array}{l}1 \\ 0\end{array}\right)+T_{\gamma}\left(\begin{array}{l}1 \\ 0\end{array}\right)$. Then by definition $f \in \gamma \mathscr{V}_{+}:$

$$
f=\gamma\left(\begin{array}{l}
u_{1} \\
u_{2}
\end{array}\right), \quad\left(\begin{array}{l}
u_{1} \\
u_{2}
\end{array}\right) \in \mathscr{V}_{+}
$$

If $f=\left(\begin{array}{l}f_{1} \\ f_{2}\end{array}\right)$, we have

$$
\sigma_{2}\left(\begin{array}{l}
f_{1}^{\sharp} \\
f_{3}^{\#}
\end{array}\right)=\sigma_{2} \gamma(z+1 / 2)\left(\begin{array}{l}
u_{1}^{\#} \\
u_{2}^{\#}
\end{array}\right)=\gamma(z) \sigma_{2}\left(\begin{array}{l}
u_{1}^{\#} \\
u_{2}^{\#}
\end{array}\right) .
$$

Hence

$$
\gamma_{f} \equiv\left(\begin{array}{rr}
f_{1} & -f_{2}^{\#} \\
f_{2} & f_{1}^{\#}
\end{array}\right)=\gamma(z)\left(\begin{array}{rr}
u_{1} & -u_{2}^{\#} \\
u_{2} & u_{1}^{\#}
\end{array}\right) .
$$

Taking determinants we obtain

$$
u_{1} u_{1}^{\#}+u_{2} u_{2}^{\#}=f_{1} f_{1}^{\#}+f_{2} f_{2}^{\#} .
$$

The LHS is holomorphic in $\Sigma_{+}$while the RHS is holomorphic on $\Sigma_{-}$except possibly for simple poles at the points at $\infty$, and each side is $K$-invariant. Hence 
each side is a constant and this is non-zero because $f_{1}=1+h$ for some $h$ which vanishes at infinity. Thus $f_{1}$ and $f_{2}$ are actually holomorphic in $\Sigma_{-}$and so Eq. (3.2) gives the desired factorisation.

Remark. In the proof we alluded to [2] and we shall now expatiate on the connection with conformal field theory. In [2] and [5] the group $\mathscr{G}_{b}$ of meromorphic functions on $\Sigma / b$ (and real analytic on $\mathscr{C} / b$ ) satisfying (2.1) was introduced. This group is isomorphic to $\mathscr{G}$ (one conjugates elements of $\mathscr{G}$ by the matrix valued function $\left(\begin{array}{cc}\lambda_{1}-\lambda_{2} & 0 \\ 0 & 1\end{array}\right)$ on $\mathscr{C}$ to produce matrix valued functions which are $b$ invariant and these descend to $\Sigma / b$ to give elements of $\mathscr{G}_{b}$.) By pulling back the constructions in [2] to $\Sigma$ we obtain an invariant bilinear form on $\mathscr{V}$ which is preserved by $\mathscr{G}$. The polarisation introduced above has $\mathscr{V}_{ \pm}$as subspaces isotropic with respect to this bilinear form and hence there is a representation of the Clifford algebra over $\mathscr{V}$ on the skew-symmetric Fock space $\Lambda \mathscr{V}_{ \pm}$. The group $\mathscr{G}$, which acts as automorphisms of the Clifford algebra, is now represented by (densely defined, unbounded) operators on the Fock space (cf. [4]). The solutions of LL described in [5] are expressed in terms of $\tau$-functions which may be interpreted in our framework as the vacuum expectation values of singular limits of elements of $\mathscr{G}$ acting on this Fock space. Closer to our present point of view, however, is the observation that solutions of LL in Lax form are constructed in [5] by elements of $\mathscr{G}$ satisfying the "bilinear identity" [5], Eq. (3.10). This bilinear identity is a consequence of Theorem 3.1.

The following is the exact analogue of the basic construction of [14, Proposition 5.13] and clearly displays the relation of Theorem 3.1 to the LL hierarchy.

Construction. Let $\Gamma_{+}$be the infinite abelian group of elements of $\mathscr{G}$ of the form

$$
G(\mathbf{t}, z)=\exp \left[i\left(\sum_{k \geqq 0} t_{2 k+1} \lambda_{3}^{2 k+1}+\sum_{k \geqq 1} t_{2 k} \lambda_{1} \lambda_{2} \lambda_{3}^{2 k-2}\right) \sigma_{3}\right] .
$$

Here we have written $\mathbf{t}$ for the infinitely many variables $\left(t_{1}=x, t_{2}, t_{3}, \ldots\right)$. Thus $\Gamma_{+}$ acts upon $\mathscr{G}$ by the formula

$$
\gamma(z) \mapsto \gamma(z) G(\mathbf{t}, z)^{-1} .
$$

For generic $\mathbf{t}$, we may perform the factorisation

$$
\gamma(z) G(\mathbf{t}, z)^{-1}=\gamma_{+}^{-1}(\mathbf{t}, z) \gamma_{-}(\mathbf{t}, z)
$$

according to Theorem 3.1. Set

$$
\Psi(\mathbf{t}, z)=\gamma_{-}(\mathbf{t}, z) G(\mathbf{t}, z)=\gamma_{+}(\mathbf{t}, z) \gamma(z) .
$$

For the differentiated action, we compute

$$
\partial_{r} \Psi \cdot \Psi^{-1}=\partial_{r} \gamma_{-} \cdot \gamma_{-}^{-1}+\gamma_{-}\left(\partial_{r} G \cdot G^{-1}\right) \gamma_{-}^{-1}=\partial_{r} \gamma_{+} \cdot \gamma_{+}^{-1} \text {. }
$$

Now

$$
\partial_{2 k+1} G \cdot G^{-1}=\lambda_{3}^{2 k+1} i \sigma_{3}, \quad \partial_{2 k} G \cdot G^{-1}=\lambda_{1} \lambda_{2} \lambda_{3}^{2 k-2} i \sigma_{3},
$$

so comparing the two expressions for the LHS of (3.7) we find that there is a trace-free $K$-equivariant matrix $B_{r}$ which is polynomial of degree $r$ in the $\lambda_{i}$ such that

$$
\partial_{r} \Psi=B_{r} \Psi
$$


The consistency conditions for these linear evolution equations is precisely the LL hierarchy as defined in Sect. 2.2.

Thus the action of $\Gamma_{+}$on the space of $\mathscr{G}_{+}$-orbits induces the LL-flows. By identifying the space of these orbits with $\mathscr{G}_{-}$(Theorem 3.1 again), we obtain:

Corollary 3.2. The map $\mathscr{G}_{-} \rightarrow \mathscr{S}$ given by

$$
\gamma \mapsto\left(\gamma_{-} \cdot i \lambda_{3} \sigma_{3} \cdot \gamma_{-}^{-1}\right)_{+}
$$

has the property that the action (3.5) of $\Gamma_{+}$on $\mathscr{G}$ goes over to the LL-flow on $\mathscr{S}$.

Remark. This map is not surjective. Roughly speaking, its image corresponds to the solitonic solutions.

\section{Invariant Holomorphic Vector Bundles over an Elliptic Curve}

In this section we prove a classification theorem for $\tilde{K}$-equivariant holomorphic $S L_{2}(\mathbb{C})$-bundles over our elliptic curve $\Sigma$. To be more precise, we begin with the

Definition. The holomorphic $S L_{2}(\mathbb{C})$-bundle $E \rightarrow \Sigma$ is said to be $\tilde{K}$-equivariant if $\tilde{K}$ acts holomorphically on $E$ in such a way as to cover the action of $K$ on $\Sigma$. Such a covering action is said to be irreducible if it preserves no proper line-subbundle of $E$.

In terms of this definition, we can state the main result of this section.

Theorem 4.1. Suppose that the holomorphic $S L_{2}(\mathbb{C})$-bundle $E \rightarrow \Sigma$ admits an irreducible covering action of $\tilde{K}$. Then $E$ is a direct sum of spin bundles $E=S \oplus S$.

The proof exploits the fact that all holomorphic bundles $E \rightarrow \Sigma$ have been classified by Atiyah [1]. Given this classification, it remains to analyze which of the available bundles can admit irreducible $\widetilde{K}$-actions.

Before giving the proof, we note that this theorem implies Theorem 3.1, at least for real-analytic elements of $\mathscr{G}$, as follows. Define an action of $\tilde{K}$ on the trivial bundles $\mathbb{C}^{2} \times \Sigma_{ \pm}$by letting $\#$ act on a vector-valued function $v$ as $\sigma_{2} v(z+1 / 2)$ and by letting $b$ act as $\sigma_{3} v(z+\tau / 2)$. If $\gamma$ lies in $\mathscr{G}$ this action patches together to the holomorphic $S L_{2}(\mathbb{C})$-bundle $E_{\gamma}$ obtained by attaching these two trivial bundles with $\gamma$. Thus $E_{\gamma}$ is an irreducible $\tilde{K}$-equivariant bundle over $\Sigma$ and so Theorem 4.1 asserts the existence of a bundle isomorphism $E_{\gamma} \simeq S \oplus S$, where $S$ is a spin bundle. By Proposition 4.3 below, $S$ is trivial and the above-mentioned bundle isomorphism gives the required factorisation.

Another corollary, relevant to the approach proposed in [13], is the following.

Corollary 4.2. Consider the $K$-invariant set $\mathscr{C}^{\prime}$ on $\Sigma$ represented on $\mathbb{C}$ by the lines $z=k \tau / 2+t$, where $t \in \mathbb{R}$ and $k \in \mathbb{Z}$. Let $\gamma: \mathscr{C}^{\prime} \rightarrow S L_{2}(\mathbb{C})$ be a $K$-equivariant loop. Then $\gamma$ factorises, $\gamma=\gamma_{+}^{-1} \gamma_{-}$, where $\gamma_{ \pm} \in \mathscr{G}_{ \pm}^{\prime}$, and $\mathscr{G}_{ \pm}^{\prime}$ have the obvious meanings.

Proof. This is essentially the same as the previous corollary. The $K$-equivariance of $\gamma$ once again guarantees that when used as a clutching function, an irreducible $\tilde{K}$-equivariant bundle is obtained.

Proposition 4.3. Let $\gamma$ be a real-analytic element of $\mathscr{G}$, and suppose that $E_{\gamma}$ is isomorphic to $S \oplus S$, where $S$ is a spin-bundle. Then $S$ is the trivial spin-bundle. 
Proof. By hypothesis, there exists a holomorphic frame $F_{ \pm}$for $S \oplus S$ over $\Sigma_{ \pm}$such that $\gamma=F_{+}^{-1} F_{-}$on $\mathscr{C}$. Because $S$ is a spin-bundle, we have

$$
F_{ \pm}(z+1)=\eta F_{ \pm}(z), \quad F_{ \pm}(z+\tau)=\zeta F_{ \pm}(z),
$$

where $\eta$ and $\zeta$ are \pm 1 . The $K$-equivariance of $\gamma$ gives

$$
\sigma_{2} \gamma(z) \sigma_{2}^{-1}=F_{+}(z+1 / 2)^{-1} F_{-}(z+1 / 2)=\sigma_{2} F_{+}(z)^{-1} F_{-}(z) \sigma_{2}^{-1},
$$

and so

$$
F_{-}(z+1 / 2) \sigma_{2} F_{-}(z)^{-1}=F_{+}(z+1 / 2) \sigma_{2} F_{+}(z)^{-1} .
$$

The LHS is a holomorphic matrix function on $\Sigma_{-}$, the RHS is holomorphic on $\Sigma_{+}$, and so each side is a constant $T_{2}$, say. Thus

$$
F_{ \pm}(z+1 / 2)=T_{2} F_{ \pm}(z) \sigma_{2}^{-1}
$$

Similarly,

$$
F_{ \pm}(z+1 / 2)=T_{3} F_{ \pm}(z) \sigma_{3}^{-1} .
$$

Moreover, det $F_{+}=\operatorname{det} F_{-}=$a constant, because det $\gamma=1$. Combining (4.1) with (4.2) and (4.3), we find that

$$
T_{2}^{2}=\eta, \quad T_{3}^{2}=\zeta, \quad T_{2} T_{3}=-T_{3} T_{2}
$$

and

$$
\operatorname{det} T_{2}=\operatorname{det} T_{3}=-1 \text {. }
$$

Now it is simple to check that if the $2 \times 2$ matrix $T$ satisfies

$$
T^{2}=-1, \quad \operatorname{det} T=-1
$$

then $T$ is $\pm i$ times the identity. So if either of $\eta$ or $\zeta$ equal -1 , the three relations (4.4) cannot be satisfied. Hence $\eta=\zeta=1$ and $S$ is the trivial spin-bundle.

The remaining subsections of this section supply the relevant background and the proof of Theorem 4.1.

4.1. Vector Bundles over $\Sigma$. We shall study bundles over $\Sigma$ by thinking of them as twisted quotients of the form $V \times \mathbb{C} / \sim$ where $V$ is some vector space and

$$
(v, z) \sim\left(v^{\prime}, z^{\prime}\right)
$$

iff $z^{\prime}=z+\lambda$ for some $\lambda \in \Lambda$ and $v^{\prime}=f(z, \lambda) v$. One checks that the automorphism $f$ has to satisfy the following conditions:

$$
f(z, 0)=1
$$

and

$$
f(z, \lambda+\mu)=f(z+\lambda, \mu) f(z, \lambda)=f(z+\mu, \lambda) f(z, \mu) .
$$

It turns out that one can always take $f(z, 1)=1$. Let us put $f(z, \tau)=A(z)$ and refer to $A$ as a (matrix) factor of automorphy. Then the above compatibility conditions give

$$
A(z+1)=A(z)
$$


We note that $A$ and $A^{\prime}$ determine isomorphic bundles iff there exists an automorphism $g(z)$ such that $g(z+1)=g(z)$ and $A^{\prime}(z) g(z)=g(z+\tau) A(z)$. In particular, if $g$ satisfies the conditions

$$
g(z+1)=g(z), \quad A(z) g(z)=g(z+\tau) A(z)
$$

then $g$ represents an automorphism of the bundle represented by the factor of automorphy $A$.

The classification of vector bundles of ranks 1 and 2 is contained in the following results.

Theorem 4.4. If $L \rightarrow \Sigma$ is a holomorphic line-bundle, then $L$ is isomorphic to a linebundle obtained from the factor of automorphy $\mathbf{e}(-w-k z)$, where $w \in \Sigma$ and $k \in \mathbb{Z}$.

The proof can be found, for example, in [10], p. 20 (the "theorem of AppellHumbert"). We remark that the integer $k$ is precisely the degree of $L$.

Theorem 4.5. If $E$ is a holomorphic $S L_{2}(\mathbb{C})$-bundle over $\Sigma$, then either $E$ is isomorphic to a direct sum of line-bundles $L \oplus L^{-1}$, or $E$ is indecomposable. In the first case $E$ can be given by a factor of automorphy of the form

$$
A(z)=\left[\begin{array}{cc}
\mathbf{e}(-w-k z) & 0 \\
0 & \mathbf{e}(w+k z)
\end{array}\right] .
$$

In the second case, E can be given by a factor of automorphy of the form

$$
A(z)=\left[\begin{array}{cc}
\mathbf{e}(-h) & \mathbf{e}(-h) \\
0 & \mathbf{e}(-h)
\end{array}\right],
$$

where $h$ is one of the half-periods $0,1 / 2, \tau / 2,1 / 2+\tau / 2$.

Proof. This is a restatement of Theorem 5 of [1].

Before we proceed to the proof of Theorem 4.1 in the next subsection, we remark that it is often convenient to discard the normalisation $f(z, 1)=1$ when dealing with the spin-bundles of $\Sigma$. With this normalization in place they correspond to factors of automorphy of the form $\mathbf{e}(-h)$, where $h$ is one of the four half-periods (or points at infinity) on $\Sigma$. On the other hand they also correspond to the choice

$$
f(z, 1)= \pm 1, \quad f(z, \tau)= \pm 1 .
$$

(The change of trivialization to make $f(z, 1)=-1$ is given by $g(z)=\mathbf{e}(-z / 2)$.)

4.2. Proof of the Theorem. The key point is to classify the lifts of the automorphisms $\#$ and $b$ of $\Sigma$. Now such a lift is the same thing as a bundle isomorphism $E^{\#} \rightarrow E$ (resp. $E^{b} \rightarrow E$ ), where $E^{\#}$ is the pull-back of $E$ by $\#$ and $E^{b}$ is the pull-back of $E$ by $b$. We note that if $E$ is given by the factor of automorphy $A$, then $E^{\sharp}$ (resp. $E^{b}$ ) is given by the factor of automorphy $A(z+1 / 2)$ (resp. $A(z+\tau / 2)$ ).

We now refer to Atiyah's Theorem 4.5. From this, one sees that for any $E, E^{\#}$ and $E^{b}$ are always of the form $E \otimes S$, where $S$ is a spin-bundle. So to lift $\#$ and $b$ is the same as finding a bundle isomorphism $E \otimes S \rightarrow E$.

Consider the first case given in Theorem 4.5, $E=L \oplus L^{-1}$. If $k=\operatorname{deg}(L)>0$, then an isomorphism $E \otimes S \rightarrow E$ either does not exist (if $S$ is non-trivial), or carries 
$L$ to $L$ (if $S$ is trivial). Such isomorphisms can never define an irreducible $\tilde{K}$-action on $E$.

If $k=0$, then $E^{\#}=E^{b}=E$ and to define an irreducible $\tilde{K}$-equivariant bundle, it is a necessary condition that $L \otimes L$ be trivial, that is, $L=L^{-1}=S$, a spin-bundle. We shall postpone the verification that for each spin-bundle $S, S \oplus S$ admits an irreducible $\tilde{K}$-action; meanwhile, we dispose of the other possibility appearing in Theorem 4.5, that where $E$ is indecomposable.

If $E$ is indecomposable, then $E^{\#}=E^{b}=E$ and as before, the problem is to construct automorphisms $g$ of $E$. For this, it is convenient to use the characterisation of such automorphisms given in Eq. (4.5). These give a matrix Fourier series

satisfying

$$
\varphi=\sum \varphi_{n} \mathbf{e}(n z)
$$

$$
\varphi_{n} \mathbf{e}(n \tau)=\left[\begin{array}{ll}
1 & 1 \\
0 & 1
\end{array}\right] \varphi_{n}\left[\begin{array}{rr}
1 & -1 \\
0 & 1
\end{array}\right] .
$$

It is easy to see from this that if $n \neq 0$, then $\varphi_{n}=0$ and hence that $\phi$ is constant and upper triangular. So $E$ cannot admit an irreducible $\tilde{K}$-action.

To complete the proof it remains to check that there exists an irreducible $\tilde{K}$-action on $S \oplus S$ for each of the four spin-bundles $S$. Think of $S \oplus S$ as $V \times \mathbb{C} / \sim$ where $\sim$ is generated by the conditions

$$
(v, z) \sim(\eta v, z+1) \text { and }(v, z) \sim(\zeta v, z+\tau)
$$

in which $\eta$ and $\zeta$ are equal to \pm 1 . Make $\sigma_{2} \in \tilde{K}$ act upon $S \oplus S$ as $(v, z) \mapsto$ $\left(\eta^{1 / 2} \sigma_{2} v, z+1 / 2\right)$ and make $\sigma_{3}$ act as $(v, z) \mapsto\left(\zeta^{1 / 2} \sigma_{3} v, z+\tau / 2\right)$. Then it is easy to check that this defines an irreducible $\tilde{K}$-action on $S \oplus S$.

\section{The Twistor Description}

5.1. Outline of the Ward Transform. The setting for the Ward transform is a holomorphic correspondence between complex manifolds $X$ (the "space-time") and $Z$ (the "twistor space"). Such a correspondence is given by a certain closed submanifold $Y$ of the product $X \times Z$. The Ward transform goes between a class of holomorphic vector bundles on $Z$ and holomorphic solutions of a non-linear PDE on $X$.

One should emphasise a few general features of the Ward transform. First, it is natural in the sense that the solution of the PDEs on $X$ depends only upon the bundle $E$ up to holomorphic equivalence. Secondly, the transform is local in $X$. In particular, no boundary conditions need be imposed upon the solutions sought on $X$; and so when applied to integrable PDEs, the Ward transform provides a tool to unify the various classes of solutions (solitonic, scattering, rapidly decreasing, etc.) that are usually described by different, albeit related, methods.

To describe the main steps in the transform, let us begin with a rank-2 holomorphic vector bundle over $Z$. In practice, $E$ will be topologically trivial and so may be identified with the product bundle, denoted $\mathbb{C}_{Z}^{2}$, over $Z$ equipped with a $\bar{\partial}$-operator $\bar{\partial}_{E}=\bar{\partial}+\alpha$, say, where $\alpha$ is a matrix-valued $(0,1)$-form on $Z$ such that the integrability condition $\bar{\partial}_{E}^{2}=0$ is satisfied.

Denote by $p$ and $q$ the natural maps $Y \rightarrow X$ and $Y \rightarrow Z$ obtained by restricting the canonical projections of $X \times Z$ to $Y$. 
5.1.1. Step $I$. Pull $E$ back to $Y$ by $q$, denoting the resulting bundle by $\tilde{E}$, say. By construction, $\tilde{E}$ is equipped with a canonical holomorphic flat relative connection. (A q-relative connection gives the means to differentiate (local) sections of $\tilde{E}$ in directions tangent to the fibres of $q$. Such a connection has a curvature form, which is an endomorphism-valued skew 2-form on the $q$-vertical tangent vectors of $Y$. A relative connection is flat if its curvature vanishes. In the case at hand, the restriction $\tilde{E} \mid q^{-1}(\zeta)$ to a fibre has a class of canonical trivializations given by pulling back frames for the fibre $E_{\zeta}$. The relative connection is uniquely defined by requiring that all connection 1 -forms vanish when the connection is written using such a trivialisation.) To be quite explicit, $\tilde{E}$ may be identified with the product bundle $\mathbb{C}_{Y}^{2}$, equipped with the $\bar{\partial}$-operator $\bar{\partial}_{\tilde{E}}=\bar{\partial}+\tilde{\alpha}\left(\tilde{\alpha}=q^{*}(\alpha)\right)$. In this gauge, $\bar{\partial}_{\tilde{E}}$ clearly commutes with any holomorphic vector field $V$ that is $q$-vertical (i.e. that is everywhere tangent to the fibres of $q$ ), and so the components of the flat relative connection are given precisely by such vector fields (in this gauge).

5.1.2. Step II. Push $\tilde{E}$ down to $X$, keeping track of the information contained in the flat relative connection. In practical terms, this is achieved by a change of gauge $g$ on $\mathbb{C}_{Y}^{2}$ from the $q$-adapted gauge of Step I to a $p$-adapted gauge. For this it is necessary to make a basic assumption about $E$ : for each point $x$ in $X$, the restriction of $\tilde{E}$ to $p^{-1}(x)$ is holomorphically trivial. Under such an assumption, together with the technical condition that $X$ is Stein, there exists a gauge transformation $g$ such that $g \cdot \bar{\partial} \cdot g^{-1}=\bar{\partial}_{\tilde{E}}$. If $V$ is any holomorphic $q$-vertical vector field on $Y$, define the operator $\hat{V}=g^{-1} \cdot V \cdot g$. Since $V$ commutes with $\bar{\partial}_{\tilde{E}}$, so $\hat{V}$ commutes with $\bar{\partial}-$ that is, $\widehat{V}$ is holomorphic. A compactness assumption on the fibres of $p$ will give a rather "mild" (roughly speaking, polynomial) dependence of $\hat{V}$ in the $p$-vertical direction. The operator $\hat{V}$ will commute with $\hat{V}^{\prime}$ whenever $V$ and $V^{\prime}$ are commuting $q$-vertical holomorphic vector fields so a commuting system of differential operators is obtained on $X$ : it is the commutativity of this system that yields the non-linear PDEs on $X$.

5.1.3. Remarks. In the case of interest, the basic set-up described above is enriched by two group actions, one of $K$ and one of $\mathbb{C}$. These have to be carried along through the above steps, and this makes for a little additional work.

As indicated at the beginning of this section, the Ward transform is natural. However we broke the gauge invariance in our brief description of Steps I and II. In fact, appropriate gauge choices can greatly facilitate the identification of the PDEs on $X$ and we shall use such choices in the rest of this section. We have not thought it appropriate to prove the existence of gauges with the described properties in this paper, as these can be relatively technical problems in complex analysis. Thus the proofs of a number of propositions in this section are omitted. However, such gauges can be defined rather explicitly for LL-bundles which arise from elements of $\mathscr{G}$ (cf. Theorem 5.3 below).

5.2. The LL Correspondence. The relevant correspondence for LL is as follows. The twistor space $Z$ is the total space of the bundle $L^{\otimes 2}$ over $\Sigma$ given by the factor of automorphy $\mathbf{e}(-8 z)$. The space-time $X$ is the space of sections of $Z$ and is biholomorphic to $\mathbb{C}^{8}$. The correspondence space $Y$ is the submanifold of $X \times Z$ of points $(x, \zeta)$ for which the point $\zeta$ of $Z$ lies on the section $x$. Notice that $Y$ can be identified with $X \times \Sigma$ so that $p$ becomes projection on the first factor. 
5.2.1. The Action of $K$. The action of $K$ is lifted to $Z$ as follows:

$$
\#:(v, z) \mapsto(-v, z+1 / 2), \quad b:(v, z) \mapsto(\mathbf{e}(\tau-4 z) v, z+\tau / 2) .
$$

We shall comment on other possible lifts of the $K$-action at the end of this section.

The given action induces one upon the space $X$ of sections of $Z$, and so too upon $Y$.

5.2.2. Coordinates on $X, Y, Z$. To introduce coordinates on these complex manifolds, we begin with a choice of basis of sections of $Z$. Put

$$
L_{0}(z)=\mathbf{e}(2 z) \vartheta_{11}(2 z), \quad L_{i}=L_{0} \lambda_{i} \quad \text { for } i=1,2,3 .
$$

These form a basis of the space of holomorphic sections of $L$. Taking into account Riemann's identities (2.10), we note that any holomorphic section of $Z$ may be written in the form

$$
v=w_{1} L_{0}^{2}+w_{2} L_{3}^{2}+\sum_{1}^{3}\left(u_{i} L_{0} L_{i}+v_{i} L_{1} L_{2} L_{3} / L_{i}\right) .
$$

In this way, the 8 numbers $\left(w_{s}, u_{i}, v_{i}\right)$ become coordinates on $X$. The following table summarises the behaviour of the coordinates on $X$ under $\#$ and $b$.

\begin{tabular}{rrrrrrrrr}
\hline & $w_{1}$ & $w_{2}$ & $u_{1}$ & $u_{2}$ & $u_{3}$ & $v_{1}$ & $v_{2}$ & $v_{3}$ \\
\hline$\#$ & $-w_{1}$ & $-w_{2}$ & $u_{1}$ & $-u_{2}$ & $u_{3}$ & $v_{1}$ & $-v_{2}$ & $v_{3}$ \\
$b$ & $w_{1}$ & $w_{2}$ & $-u_{1}$ & $-u_{2}$ & $u_{3}$ & $-v_{1}$ & $-v_{2}$ & $v_{3}$ \\
\hline
\end{tabular}

In what follows we shall be mainly concerned with the following restriction of the correspondence. Put $X_{0}=\left\{w_{2}=u_{1}=u_{2}=v_{1}=v_{2}=0\right\}$ and $Y_{0}=p^{-1}\left(X_{0}\right)$. Then the restriction of $q$ to $Y_{0}$ maps onto $Z$.

Coordinates on $Z$ may be introduced by putting

$$
(v, z)=\left(L_{0}^{2} \mu, z\right) \text { away from } \infty
$$

and

$$
(v, z)=\left(L_{3}^{2} \mu^{\prime}, z\right) \text { near } \infty .
$$

If $Z_{ \pm}$denotes the part of $Z$ which lies over $\Sigma_{ \pm}$, then $\mu$ is a fibre coordinate on $Z_{+}, \mu^{\prime}$ is a fibre coordinate defined over $Z_{-}$and $\mu=\lambda_{3}^{2} \mu^{\prime}$ over $Z_{+} \cap Z_{-}$.

From (5.1), the following are $q$-vertical holomorphic vector fields on $Y_{0}$ :

$$
U=L_{0} \partial / \partial u_{3}-L_{3} \partial / \partial w_{1}, \quad V=L_{0}^{2} \partial / \partial v_{3}-L_{1} L_{2} \partial / \partial w_{1}
$$

These form a commuting basis of the $q$-vertical tangent space at each point of the finite part of $Y_{0}$ (i.e. the part where $L_{0} \neq 0$ ). Strictly, and this will be important later, $U$ and $V$ are vector fields with values in $L$ and $L^{\otimes 2}$ respectively. For the present, we shall continue to call them vector fields.

5.2.3. The $\mathbb{C}$-Action. We define an action of $\mathbb{C}$ on $X$ by translation of the $w_{1}$ coordinate. The corresponding action on $Z$ is:

$$
(v, z) \mapsto\left(v+L_{0}^{2} s, z\right) \quad(s \in \mathbb{C}) .
$$


In particular, this action fixes the points at $\infty$ on $Z$. The action is generated by the vector field $\partial / \partial w_{1}$ on $X$ and by $\xi=L_{0}^{2} \partial / \partial v$ on $Z$. In coordinates over the finite part of $Z, \xi$ is $\partial / \partial \mu$. The induced action on $Y$ is generated by $\widetilde{\xi}=\partial / \partial w_{1}$; indeed,

$$
q_{*}(\tilde{\xi})=\xi \text {. }
$$

5.3. LL-Bundles over $Z$. A holomorphic $\mathrm{SL}_{2}(\mathbb{C})$-bundle $E \rightarrow Z$ is called a LandauLifshitz (or LL) bundle if:

1. $E$ is irreducible $\tilde{K}$-equivariant;

2. $E$ admits a holomorphic $\mathbb{C}$-action covering that on $Z$ described in Sect. 5.2.3, acting by $\exp \left(i s \sigma_{3}\right)$ on the fibres at $\infty$ and compatible with the $\tilde{K}$-action;

3. the restriction of $E$ to each section of $Z$ is holomorphically trivial.

Remark. The notion of $\tilde{K}$-equivariance was defined in Sect. 4 only for bundles over $\Sigma$. But we have already defined the action of $K$ on $Z$ so the meaning of Property 1 is clear.

The Ward transform we are going to describe in this section begins with an LL-bundle over $Z$ and produces a holomorphic solution of LL on the 2-dimensional space $X_{0} / \mathbb{C}$, where the $\mathbb{C}$-action on $X$ has been defined in Sect. 5.2.3. To facilitate the description it is convenient to have the data of the LL-bundle presented in a standard way. To do that, start from the observation that any $\mathrm{SL}_{2}(\mathbb{C})$-bundle $E \rightarrow Z$ is topologically trivial. So we can choose a smooth identification of $E$ with the product bundle $\mathbb{C}_{Z}^{2}$ on $Z$ and we claim that this can be chosen in such a way that the $\tilde{K}$-action on $E$ is given by

$$
\#:(v, z ; e) \mapsto\left(-v, z+1 / 2, \sigma_{2} e\right) \quad b:(v, z ; e) \mapsto\left(\mathbf{e}(\tau-4 z), z+\tau / 2, \sigma_{3} e\right),
$$

where $e \in \mathbb{C}^{2}$ is the fibre variable $\mathbb{C}_{Z}^{2}$. Having done this, the holomorphic structure on $E$ is completely determined by a $\bar{\partial}$-operator $\bar{\partial}_{E}=\bar{\partial}+\alpha$, where $\alpha$ is a matrix valued $(0,1)$-form on $Z$. In such a trivialisation, $\alpha$ satisfies the following conditions:

$$
\operatorname{tr}(\alpha)=0
$$

(because $E$ is an $\mathrm{SL}_{2}(\mathbb{C})$-bundle) and

$$
\alpha^{\sharp}=\sigma_{2} \alpha \sigma_{2}^{-1}, \quad \alpha^{b}=\sigma_{3} \alpha \sigma_{3}^{-1},
$$

where $\alpha^{\#}$ (resp. $\left.\alpha^{b}\right)$ is the pull-back of $\alpha$ by $\#$ (resp. by b). By choosing a $K$ equivariant holomorphic frame near $\infty$ and by using the fact that the fibres of $Z$ are Stein we may prove the following:

Proposition 5.1. If $E$ is a LL-bundle over $Z$, a smooth trivialisation $E \rightarrow \mathbb{C}_{Z}^{2}$ can be chosen so that

1. the $\bar{\partial}$-operator of $E$ takes the form $\bar{\partial}_{E}=\bar{\partial}+\alpha d \bar{z}$;

2. the matrix function $\alpha$ is trace-free and satisfies $\alpha^{\#}=\sigma_{2} \alpha \sigma_{2}^{-1}, \alpha^{b}=\sigma_{3} \alpha \sigma_{3}^{-1}$;

3. there is a K-equivariant open neighbourhood $W$ of $\infty$ such that $\alpha=0$ in $W$.

To take into account the $\mathbb{C}$-action on $E$, we work with its differentiated version, which is given by a Lie derivative operator $\mathscr{L}_{\xi}$ on $E$. In the gauge of Proposition 5.1, $\mathscr{L}_{\xi}$ has the form $\mathscr{L}_{\xi}=\xi-\beta$, where $\beta$ is some smooth trace-free matrix with $\beta(\infty)=i \sigma_{3}$. The properties of $\beta$ that correspond to the $\mathbb{C}$-action being holomorphic and compatible with the $\tilde{K}$-action are summarised as follows: 
Proposition 5.2. If $E$ is a LL-bundle over $Z$, trivialised as in Proposition 5.1, then $\mathscr{L}_{\xi}=\xi-\beta$ satisfies:

1. $\left[\bar{\partial}_{E}, \mathscr{L}_{\xi}\right]=0$

2. $\beta^{\ddagger}=-\sigma_{2} \beta \sigma_{2}^{-1}, \beta^{b}=\sigma_{3} \beta \sigma_{3}^{-1}$;

3. $\beta$ is holomorphic in $W$.

Moreover, given any integer $N>0$, there is a change of holomorphic gauge in $W$ which gives $\beta=i \sigma_{3}+O\left(\lambda_{3}^{-N}\right)$ in $W$.

Proof. All except the last part follow directly from the definitions. That part is obtained by the argument of Theorem 2.3 (with the role of $x$ taken here by $\lambda_{3} \mu$ ), truncated after $N$ steps.

Remark. It may be helpful to point out that if $\beta$ takes a constant value at $\infty$ in $Z$, then the $K$-equivariance statement of this proposition forces $\beta$ to be a multiple of $\sigma_{3}$.

There is an important class of examples of LL-bundles for which one can choose $\beta$ identically equal to $i \sigma_{3}$ in $W$, our $K$-invariant neighbourhood of $\infty$. These correspond to the solitonic solutions constructed at the end of Sect. 3 .

Theorem 5.3. There is a natural map from $\mathscr{G}_{\text {_ }}$ to the class of LL-bundles.

Proof. If $\gamma \in \mathscr{G}_{-}$, consider the holomorphic bundle $E$ over $Z$ obtained from the clutching function

$$
P(\mu, z)=\gamma(z) \exp \left(-i \mu \sigma_{3}\right) \text { on } Z_{+} \cap Z_{-} .
$$

In this description, $\mathscr{L}_{\xi}$ is given by $\partial_{\mu}$ on $Z_{+}$and by $\partial_{\mu}-i \sigma_{3}$ on $Z_{-}$. To bring this description into coincidence with that of Proposition 5.1, use a bump function to construct a smooth $K$-equivariant modification $\gamma^{\prime}$ of $\gamma$ which agrees with $\gamma$ near $\infty$, but which equals the identity outside a neighbourhood of $\Sigma_{-}$. Then if $\left(s_{+}, s_{-}\right)$is a smooth section of $E$,

$$
e=\left\{\begin{aligned}
\left(\gamma^{\prime} \exp \left(-i \mu \sigma_{3}\right)\right)^{-1} s_{+} & \text {on } Z_{+} ; \\
s_{-} & \text {on } Z_{-} ;
\end{aligned}\right.
$$

becomes a smooth section of $\mathbb{C}_{Z}^{2}$. Then

$$
\alpha=\exp \left(i \mu \sigma_{3}\right) \gamma^{\prime-1} \partial_{\bar{z}} \gamma^{\prime} \exp \left(-i \mu \sigma_{3}\right)
$$

is supported in $\Sigma_{+}$and $\beta=i \sigma_{3}$ identically over all of $Z$. The triviality of $E$ over any section of $Z$ is guaranteed by Theorem 4.1 .

Remark. The map is "natural" in the sense that the Ward transform restricts to give the construction described at the end of Sect. 3. The truth of this claim should be apparent by the end of this section.

5.4. The Ward Transform. Recall that Step I of the Ward transform pulls $E$ back to $Y$. In fact, we restrict to the subspace $Y_{0}$ of $Y$ defined in Sect. 5.2.2. With coordinates $(x, z)=\left(w_{1}, u_{3}, v_{3}, z\right)$ on $Y_{0}$ we can summarise the result of applying this step to a LL bundle presented according to Propositions 5.1 and 5.2 as follows.

Proposition 5.4. The pull-back to $Y_{0}$ of a LL bundle is equivalent to the (gauge equivalence class of) following data: a $\bar{\partial}$-operator $\bar{\partial}_{\tilde{E}}=\bar{\partial}+\tilde{\alpha} d \bar{z}$ and a symmetry 
operator $\mathscr{L}_{\tilde{\xi}}=\partial_{w_{1}}-\tilde{\beta}$ on the product bundle $\mathbb{C}_{Y_{0}}^{2}$ over $Y_{0}$ which satisfy the following conditions:

1. K-equivariance: $\tilde{\alpha}^{\#}=\sigma_{2} \tilde{\alpha} \sigma_{2}^{-1}, \tilde{\alpha}^{b}=\sigma_{3} \tilde{\alpha} \sigma_{3}^{-1} ; \widetilde{\beta}^{\sharp}=-\sigma_{2} \tilde{\beta} \sigma_{2}^{-1}, \widetilde{\beta}^{b}=\sigma_{3} \tilde{\beta} \sigma_{3}^{-1}$;

2. compatibility: $\left[\bar{\partial}_{\tilde{E}}, \mathscr{L}_{\tilde{\xi}}\right]=0$;

3. relative flatness: $\left[\bar{\partial}_{\tilde{E}}, V\right]=0,\left[\mathscr{L}_{\tilde{\xi}}, V\right]=0$ for any holomorphic q-vertical vector field $V$;

4. the asymptotic conditions: $\tilde{\alpha}=0, \tilde{\beta}=i \sigma_{3}+O\left(\lambda_{3}^{-N}\right)$ in a neighbourhood $W$ of $\infty$ for some integer $N>0$.

Proof. Take $\alpha$ and $\beta$ as in Propositions 5.1 and 5.2 and put $\tilde{\alpha}=q^{*}(\alpha), \tilde{\beta}=q^{*}(\beta)$. It is then straightforward to check the conditions listed in the proposition.

Step II of the Ward transform, push-down to $X$, is accomplished in practical terms by finding the gauge transformation $g$ on $Y_{0}$ which intertwines $\bar{\partial}_{\tilde{E}}$ and $\bar{\partial}$. Such a $g$ exists because of the basic triviality assumption about LL-bundles. In fact, one can insist that $g$ satisfy all the conditions of the following proposition:

Proposition 5.5. Given the pull-back $\tilde{E}$ of a LL-bundle on $Y_{0}$, presented according to Proposition 5.4 one can find a gauge transformation $g: \mathbb{C}_{Y_{0}}^{2} \rightarrow \mathbb{C}_{Y_{0}}^{2}$ such that:

1. $g \cdot \bar{\partial}_{\tilde{E}} \cdot g^{-1}=\bar{\partial}$;

2. $g \cdot \mathscr{L}_{\tilde{\xi}} \cdot g^{-1}=\tilde{\xi}=\partial_{w_{1}}$;

3. $g$ is K-equivariant: $g^{\sharp}=\sigma_{2} g \sigma_{2}^{-1}$ and $g^{b}=\sigma_{3} g \sigma_{3}^{-1}$;

4. near $\infty, g$ is holomorphic.

Proof. Because $\bar{\partial}_{\tilde{E}}$ and $\mathscr{L}_{\tilde{\xi}}$ commute, one can find a gauge transformation $g$ which satisfies the first two conditions. Any such $g$ will also satisfy the fourth condition (because of the corresponding condition of the previous Proposition). To analyze the $\tilde{K}$-equivariance, put $T_{2}=\left(g^{\sharp}\right)^{-1} \sigma_{2} g$ and $T_{3}=\left(g^{b}\right)^{-1} \sigma_{3} g$.

By pulling back the first two conditions by $\#$ and $b$ and using the $\tilde{K}$ equivariance of $\alpha$ and $\beta$ we find that $T_{2}$ and $T_{3}$ are holomorphic and independent of $w_{1}$. The only globally defined holomorphic functions on $\Sigma$ are the constants, so in fact $T_{2}$ and $T_{3}$ are independent of $z$. By definition of the $K$-action on $Y_{0}$, it follows at once that

$$
T_{2}^{\#}=T_{2}^{b}=T_{2}
$$

and similarly for $T_{3}$. Thus, as in the proof of Proposition 4.3,

$$
T_{2}^{2}=T_{3}^{2}=1, \quad T_{2} T_{3}=-T_{3} T_{2} .
$$

So by the representation theory of $\tilde{K}$, there is an invertible matrix $Q$ such that $T_{2}=Q \sigma_{2} Q^{-1}$ and $T_{3}=Q \sigma_{3} Q^{-1}$. Replacing $g$ by $g \cdot Q$ yields a gauge transformation which has all the required properties.

Now with such a $g$ chosen, put $\hat{U}=g \cdot U \cdot g^{-1}$ and $\hat{V}=g \cdot V \cdot g^{-1}$, where $U$ and $V$ are the vertical vector fields of (5.2). Because they commute with $\bar{\partial}$ they are holomorphic operators on $Y_{0}$ and because $U$ has values in $L$ while $V$ has values in $L^{2}$, they are polynomial in the $L_{i}$ of respective degrees 1 and 2 . Written explicitly,

$$
\begin{aligned}
& \hat{U}=g \cdot\left(L_{0} \partial_{u_{3}}-L_{3} \partial_{w_{1}}\right) \cdot g^{-1}, \\
& \hat{V}=g \cdot\left(L_{0}^{2} \partial_{v_{3}}-L_{1} L_{2} \partial_{w_{1}}\right) \cdot g^{-1},
\end{aligned}
$$


and

$$
g \cdot \partial_{w_{1}} \cdot g^{-1}=\partial_{w_{1}}+g \beta g^{-1}
$$

Use (5.9) to write the operators (5.7) and (5.8) in the form

$$
\begin{aligned}
& \hat{U}=g \cdot\left(L_{0} \partial_{u_{3}}-L_{3} \beta\right) \cdot g^{-1}+L_{3} \partial_{w_{1}}, \\
& \hat{V}=g \cdot\left(L_{0}^{2} \partial_{v_{3}}-L_{1} L_{2} \beta\right) \cdot g^{-1}+L_{1} L_{2} \partial_{w_{1}} .
\end{aligned}
$$

Now divide by the $\mathbb{C}$-action; on invariant sections, $\partial_{w_{1}}$ acts by zero, and so $\hat{U}$ and $\hat{V}$ take the form of a LL Lax pair, by the truncated version of Theorem 2.3.

This observation completes our long journey from a LL bundle on $Z$ to a holomorphic solution of LL on the invariant slice of $X$.

5.5. Remarks. The inverse construction is achieved by reversing the steps described above. The details are omitted, save to point out that given a solution of LL in the form of the Lax pair $\hat{U}, \hat{V}$, the fibre at $\zeta \in Z$ of the corresponding holomorphic bundle is given by

$$
E_{\zeta}=\{s: \hat{U} s=\hat{V} s=0\} .
$$

There are two simple generalisations of the twistor description which require very little work to set up. The first concerns the twistor description of local solutions. One can restrict the correspondence between $X$ and $Z$ to an open subset $U$ of $X$; provided this is, say, convex, everything in this section will go through to give a correspondence between LL bundles on the corresponding region of $Z$ and LL solutions on $U$.

The second generalisation concerns the hierarchies. The "higher flows" of the LL hierarchy can be incorporated by considering LL bundles over the manifold $Z_{N}$, which is by definition the total space of $L^{\otimes N}$ over $\Sigma$.

A final remark concerns the choice of lift of the action of $K$ to $Z$. A different such choice will result in a different 2-dimensional subspace of $X$ being fixed by the action. Our choice corresponds to the particular presentation of LL which singled out $\lambda_{3}$ over $\lambda_{1}$ and $\lambda_{2}$. Other choices correspond, in essence, to permutations of the $\lambda_{i}$.

\section{A. Appendix: Frechet Grassmannians}

Other work [2,3], on Clifford algebras (or fermions) on an elliptic curve indicates that the natural setting is a $C^{\infty}$ version of the $L^{2}$ infinite Grassmannians of [12].

This Appendix is thus devoted to a careful construction of the Grassmannian of $C^{\infty}\left(\mathbf{S}^{1}\right)$ and the corresponding restricted general linear group and to a development of their basic properties. The treatment is dictated by the requirements of the proof of Theorem 3.1 and could certainly be taken much further.

Let $V$ be a finite dimensional inner product space with an inner product (,) and norm $|\cdot|$. Denote by $\mathscr{V}$ the space of smooth $V$-valued functions on $S^{1}$. The usual Frechet topology of $\mathscr{V}$ may be defined by a countable family of Sobolev norms $\|\cdot\|_{k}$, with

$$
\|f\|_{k}^{2}=\int_{S^{1}}\left(|f(\theta)|^{2}+\left|\frac{d^{k}}{d \theta^{k}} f(\theta)\right|^{2}\right) \mathrm{d} \theta
$$


Equivalently, in terms of Fourier series:

$$
\|f\|_{k}^{2}=\sum_{n}\left(1+n^{2 k}\right)\left|f_{n}\right|^{2} .
$$

As in Nash-Moser theory, we make the following:

Definition. An operator $A$ on $\mathscr{V}$ is said to be of degree $m$ if it extends to a bounded operator $L_{k}^{2} \rightarrow L_{k+m}^{2}$ for each $k$. It is said to be compact of degree $m$ if it extends to a compact operator $L_{k}^{2} \rightarrow L_{k+m}^{2}$ for each $k$.

Since the inclusion $L_{k+1}^{2} \rightarrow L_{k}^{2}$ is compact, we have

Proposition A.1. If the operator $A$ on $\mathscr{V}$ is of degree $m$, then it is compact of degree $m+1$.

To define $\mathrm{GL}_{\text {tame }}(\mathscr{V})$, we polarise $\mathscr{V}$ in the usual way: $\mathscr{V}=\mathscr{V}_{+} \oplus \mathscr{V}$ - with $\mathscr{V}_{ \pm}$ being the subspaces of functions which have analytic extensions to the interior (resp. exterior) of the unit disc with the convention that the constants lie in $\mathscr{V}_{+}$. This done, let $Q=Q_{+}-Q_{-}$with $Q_{ \pm}$being the projections onto $\mathscr{V}_{ \pm}$.

Definition. Denote by $\mathrm{GL}_{\text {tame }}(\mathscr{V})$ the set of all invertible maps $A: \mathscr{V} \rightarrow \mathscr{V}$ such that $A$ is of degree 0 and $[Q, A]$ is of degree -1 .

We shall often write an operator $A$ on $\mathscr{V}$ as a $2 \times 2$ matrix of operators relative to the polarisation $\mathscr{V}=\mathscr{V}_{+} \oplus \mathscr{V}_{-}$,

$$
A=\left(\begin{array}{ll}
a & b \\
c & d
\end{array}\right) .
$$

Then the definition says that $A \in \mathrm{GL}_{\text {tame }}(\mathscr{V})$ if it is invertible, $a$ and $d$ are of degree 0 , and $b$ and $c$ are of degree -1 . Now we can prove

Proposition A.2. The set $\mathrm{GL}_{\text {tame }}(\mathscr{V})$ is a topological group with a natural Frechet topology.

Proof. The product of two elements of $\mathrm{GL}_{\text {tame }}(\mathscr{V})$ lies in $\mathrm{GL}_{\text {tame }}(\mathscr{V})$ because the set of operators of degree -1 is an ideal in the ring of operators of degree 0 . Suppose now that $A \in \mathrm{GL}_{\text {tame }}(\mathscr{V})$ has the block matrix representation above and that

$$
A^{-1}=\left(\begin{array}{ll}
p & q \\
r & s
\end{array}\right) \text {. }
$$

By the open mapping theorem, $A^{-1}$ is of degree 0 . So to check that $A^{-1} \in \mathrm{GL}_{\text {tame }}(\mathscr{V})$ we have only to prove that $q$ and $r$ are of degree -1 . Now.

$$
p b+q d=0 \text { and } r b+s d=1,
$$

so $q d$ is of degree -1 and $d$ is Fredholm (as an operator on $L_{k}^{2}$ ). Thus the image of $d$ is of finite codimension and it follows that $q$ is of degree -1 . Similarly, $r$ is of degree -1 .

Thus $\mathrm{GL}_{\text {tame }}(\mathscr{V})$ is a group. It can be equipped with a Frechet topology defined by using the sequence of operator norms $L_{k}^{2} \rightarrow L_{k}^{2}$ for the diagonal blocks and $L_{k}^{2} \rightarrow L_{k+1}^{2}$ for the off-diagonal blocks. The verification that the group operations are continuous in this topology is omitted.

The topology we have just defined on $\mathrm{GL}_{\text {tame }}(\mathscr{V})$ (though not the actual norms) is independent of the inner product on $V$, so we may think of $\mathrm{GL}_{\text {tame }}(\mathscr{V})$ as associated to the polarised space $\mathscr{V}$. 
We saw in the course of the proof that the diagonal blocks of $A$ are Fredholm. Because it is true for nuclear spaces that any compact perturbation of a Fredholm operator is Fredholm of the same index, we have ind $(a)=-\operatorname{ind}(d)$. As in the $L^{2}$ case, the connected components of $\mathrm{GL}_{\text {tame }}(\mathscr{V})$ are given by the index map $A \mapsto \operatorname{ind}\left(Q_{+} A Q_{+}\right)$.

Natural subgroups of $\mathrm{GL}_{\text {tame }}(\mathscr{V})$ are obtained by considering the operators which preserve an inner product on $\mathscr{V}$ and those preserving a non-degenerate bilinear form on $\mathscr{V}$. We denote these respectively by $\mathscr{U}_{\text {tame }}$ and $\mathcal{O}_{\text {tame }}$.

Let LGL $(V)$ denote the group of smooth loops in GL( $V)$. Then $\operatorname{LGL}(V)$ acts by multiplication on $\mathscr{V}$. Moreover, we have

Proposition A.3. The multiplication action of $\operatorname{LGL}(V)$ on $\mathscr{V}$ defines an embedding into $\mathrm{GL}_{\text {tame }}(\mathscr{V})$.

Proof. It is immediate that multiplication by a smooth loop is an operator of degree 0 on $\mathscr{V}$. To prove that the off-diagonal blocks are of degree -1 , let $f \in \mathscr{V}_{+}$ and $\gamma \in \operatorname{LGL}(V)$ be expanded in Fourier series:

$$
f=\sum_{m \geqq 0} f_{m} e^{i m \theta}, \quad \gamma=\sum_{m} \gamma_{m} e^{i m \theta}
$$

where the $\gamma_{m}$ are endomorphisms of $V$. Then

and

$$
(\gamma f)_{-}=\sum_{n<0}\left(\sum_{m \geqq 0} \gamma_{n-m} f_{m}\right) e^{i m \theta}
$$

$$
\begin{aligned}
& \left\|(\gamma f)_{-}\right\|_{\ell}^{2}=\sum_{n<0}\left(1+n^{2}\right)^{\ell}\left|\sum_{m \leqq 0} \gamma_{n+m} f_{-m}\right|^{2} \\
\leqq & \sum_{n<0}\left(1+n^{2}\right)^{\ell} \sum_{m \leqq 0}\left(1+m^{2}\right)^{-k}\left|\gamma_{n+m}\right|^{2}\|f\|_{k}^{2} \\
\leqq & \sum_{N<0}\left(1+N^{2}\right)^{\ell+1}\left|\gamma_{N}\right|^{2}\|f\|_{k}^{2}=\|\gamma-\|_{\ell+1}^{2}\|f\|_{k}^{2} .
\end{aligned}
$$

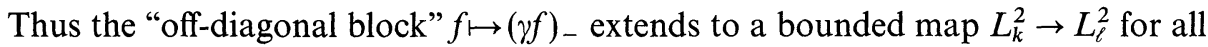
$\ell$ and $k$.

Just as in the $L^{2}$ case, the topology introduced on $\operatorname{LGL}(V)$ by restricting that of $\mathrm{GL}_{\text {tame }}(\mathscr{V})$ is much coarser than the $C^{\infty}$ topology on $\mathrm{LGL}(V)$. However it is much finer than the $L_{1 / 2}^{2}$ topology which is induced by the topology of $L^{2}$ restricted general group $\mathrm{GL}_{\text {res. }}$. This is one reason why we have taken the trouble of introducing it.

Having taken some trouble over the definition of the group $\mathrm{GL}_{\text {tame }}(\mathscr{V})$, we shall now give a rather swifter account of the corresponding restricted Grassmannian.

Definition. We denote by $\mathrm{Gr}_{\text {tame }}$ the set of all closed subspaces $\mathscr{W}$ of $\mathscr{V}$ such that the projection $P_{\mathscr{W}}: \mathscr{W} \rightarrow \mathscr{V}_{+}$is Fredholm (of degree 0 ); the projection $\mathscr{W} \rightarrow \mathscr{V}_{-}$is of degree -1 .

The proofs of the following can be found by making the obvious modifications to the proofs of the corresponding results in [12, Sect. 7.1].

Proposition A.4. $\mathrm{Gr}_{\text {tame }}$ is a Frechet manifold modelled on the Frechet space of operators from $\mathscr{V}_{+}$to $\mathscr{V}_{-}$of degree -1 . Moreover, $\mathscr{U}_{\text {tame }}$ acts transitively on $\mathrm{Gr}_{\text {tame }}$.

This statement of transitivity implies the key fact needed in the proof of Theorem 3.1, that if $\mathscr{W} \in \mathrm{Gr}_{\text {tame }}$ is transverse to $\mathscr{V}_{-}$, then there is an operator $T$ of 
degree -1 such that $\mathscr{W}$ is the graph of $T$. As explained in the proof of that Theorem, the polarised space $\mathscr{V}$ introduced there can be identified with the standard polarised space $C^{\infty}\left(S^{1}, V\right)$ (for an appropriate choice of $V$ ).

We conclude this appendix with some remarks which may assist the reader to connect the viewpoint of this paper with that of [2]. In the latter there are two crucial ingredients. The first is the existence of a distinguished bilinear form on $\mathscr{V}$ which is preserved by the functions $\mathscr{G}$. Although we do not make this explicit here it is a consequence of the $K$ equivariance. So, the group $\mathscr{G}$ of Sect. 3 is contained in the group $L \mathcal{O}=\operatorname{LGL}(V) \cap \mathcal{O}_{\text {tame }}$ of loops preserving the bilinear form on $\mathscr{V}$. This latter group leaves the connected component of $\mathscr{V}$ in the Grassmannian $\mathrm{Gr}_{\text {tame }}$ (the set of subspaces of virtual dimension zero) invariant. In fact the orbit of $L \mathcal{O}$ consists of polarisations of $\mathscr{V}$ by subspaces isotropic with respect to the bilinear form. This orbit of polarisations has two connected components (necessarily because there are just two connected components in $L \mathcal{O}$ ). These components are distinguished by the parity of the dimension of the kernel of the Fredholm operator $Q_{+} A Q_{+}$for $A \in L \mathcal{O}$.

The second ingredient in [2] is the connection with Clifford algebras and their representations which we will not go into here.

Acknowledgement. The early stages of this work were influenced by ideas of John Palmer. We thank him, and also Mike Eastwood for several useful discussions.

\section{References}

1. Atiyah, M.F.: Vector bundles over an elliptic curve. Proc. Lond. Math. Soc. 7, 414-452 (1957)

2. Carey, A.L., Eastwood, M.G., Hannabuss, K.C.: Riemann surfaces, Clifford algebras and infinite dimensional groups. Commun. Math. Phys. 130, 217-236 (1990)

3. Carey, A.L., Hannabuss, K.C., Murray, M.K.: Free fermions on Riemann surfaces and spectral curves of the Chiral Potts model. Proceedings of the 2nd International Conference on Topological Methods in Quantum Field Theory, Turku, Finland, 1991, Singapore: World Scientific 1992

4. Carey, A.L., Palmer, J.: Infinite complex spin groups. J. Funct. Anal. 83, 1-43 (1989)

5. Date, E., Jimbo, M., Kashiwara, M., T. Miwa, Y.: Landau-Lifshitz equation: Solitons, quasiperiodic solutions and infinite dimensional Lie algebras. J. Phys. A: Math. Gen. 16, 221-236 (1983)

6. Drinfeld, V.G., Sokolev, V.V.: Lie algebras and equations of Korteweg-de Vries type. Dokl. Akad. Nauk SSSR 258, 11-16 (1981)

7. Faddeev, L.D., Takhtajan, L.A.: Hamiltonian Methods in the Theory of Solitons. Berlin, Heidelberg, New York: Springer 1987

8. Mason, L.J., Sparling, G.: Twistor Correspondences for the soliton hierarchies. J. Geom. and Phys. 8, 243-272 (1992)

9. Mason, L.J., Singer, M.A.: The geometry of equations of KdV type. In preparation

10. Mumford, D.: Abelian Varieties. Oxford: Oxford University Press 1985

11. Mumford, D.: Tata Lectures on Theta I. Progress in Mathematics 28, Boston: Birkhäuser 1983

12. Pressley, A.N., Segal, G.B.: Loop Groups. Oxford: Oxford University Press 1986

13. Rodin, Y.: The Riemann boundary problem on Riemann surfaces and the inverse scattering problem for the Landau-Lifshitz equation. Physica 11D, 90-108 (1984)

14. Segal, G.B., Wilson, G.: Loop groups and equations of KdV type. Publ. Math. IHES 61, 5-65 (1985)

15. Ward, R.S.: Integrable and solvable systems and relations among them. Philos. Trans. Roy. Soc. Lond. A315, 451-457 (1985) 
